\title{
Refractory Urothelial Carcinoma
}

National Cancer Institute

\section{Source}

National Cancer Institute. Refractory Urothelial Carcinoma. NCI Thesaurus. Code C150364.

Urothelial carcinoma that does not respond to treatment. 